\title{
An eight-month-old child with cervical adenitis
}

\author{
Melanie CM Murray MD PhD¹, Jacqueline Cooper $\mathrm{MD}^{2}$, Patrick Tang MD PhD ${ }^{3,4}$, Jan Hajek $\mathrm{MD}^{1,4}$, \\ Tobias R Kollman MD PhD ${ }^{5}$
}

\section{CASE PRESENTATION}

A previously well, eight-month-old girl presented with erythematous swelling in the right submandibular region (Figure 1). According to her parents, nodular swellings had appeared three weeks previously in the temple and submandibular regions, and were increasing in size. Eleven days before presentation, the parents attended a primary care clinic where seven days of oral cephalexin was prescribed, without effect.

The patient had no constitutional symptoms, and growth and development were normal. The child had been born in Canada following an uncomplicated pregnancy and vaginal delivery, and she was breastfed exclusively for five months. Her vaccinations were up to date, and did not include Bacille Calmette-Guérin. The patient had travelled with her mother on two three-week trips to Bangkok, Thailand, when she was between four and seven months of age. Both times the family stayed with the patient's maternal grandmother, who had an undiagnosed respiratory illness (that included cough and weight loss) and in whom Mycobacterium tuberculosis (MTB) had not been excluded. During travel, the patient reportedly had no animal contacts, insect bites or water exposures, and never consumed undercooked meats, unpasteurized dairy products or tap water.

On examination, the patient was afebrile with normal vital signs. Palpation of the right-sided, anterior cervical, infraclavicular and supraclavicular lymph nodes revealed lymphadenopathy. There was a palpable, nontender right temple mass $1 \mathrm{~cm}$ in size, and a fluctuant, erythematous, nontender submandibular mass $4 \mathrm{~cm}$ in size (Figure 1). The remainder of the physical examination was noncontributory.

Laboratory investigations revealed a white blood cell count of $18.1 \times 10^{9} / \mathrm{L}$, a hemoglobin level of $119 \mathrm{~g} / \mathrm{L}$ and a platelet count of $353 \times 10^{9} / \mathrm{L}$. Electrolytes, creatinine and C-reactive protein levels were normal. Blood cultures were negative after five days incubation. A chest $\mathrm{x}$-ray was normal. An ultrasound scan demonstrated a complex, hypoechoic mass $3.7 \mathrm{~cm}$ in size, with internal septations in the subcutaneous tissue of the submandibular region associated with local adenopathy, and a $1 \mathrm{~cm}$ cystic lesion at the right temple. Tuberculin skin testing (TST), performed using the Mantoux method with five tuberculin units of purified protein derivative, was positive at $17 \mathrm{~mm}$ of induration. The patient was HIV negative, and there was no clinical suspicion of other immunodeficiencies.

On admission, the patient was started empirically on intravenous clindamycin. Due to lack of improvement on clindamycin after four days, the patient underwent surgical incision and drainage of the submandibular mass for diagnostic and therapeutic purposes.

\section{DIAGNOSIS}

Microscopic examination of surgical samples detected 1+ acid-fast bacilli. Gram and fungal stains and bacterial and fungal cultures were negative. Biopsy material underwent standard molecular testing for MTB using the Amplified M tuberculosis Direct assay (Gen-Probe, USA) and Mycobacterium avium complex (MAC) testing with the appropriate AccuProbe assay (Gen-Probe, USA). Both tests were negative. For organism identification, polymerase chain reaction analysis of the heat shock protein hsp65 was performed, yielding a $401 \mathrm{bp}$ amplicon that was sequenced (1). The hsp65 sequence identified the organism (10 days after sample collection) as Mycobacterium haemophilum. After 40 days

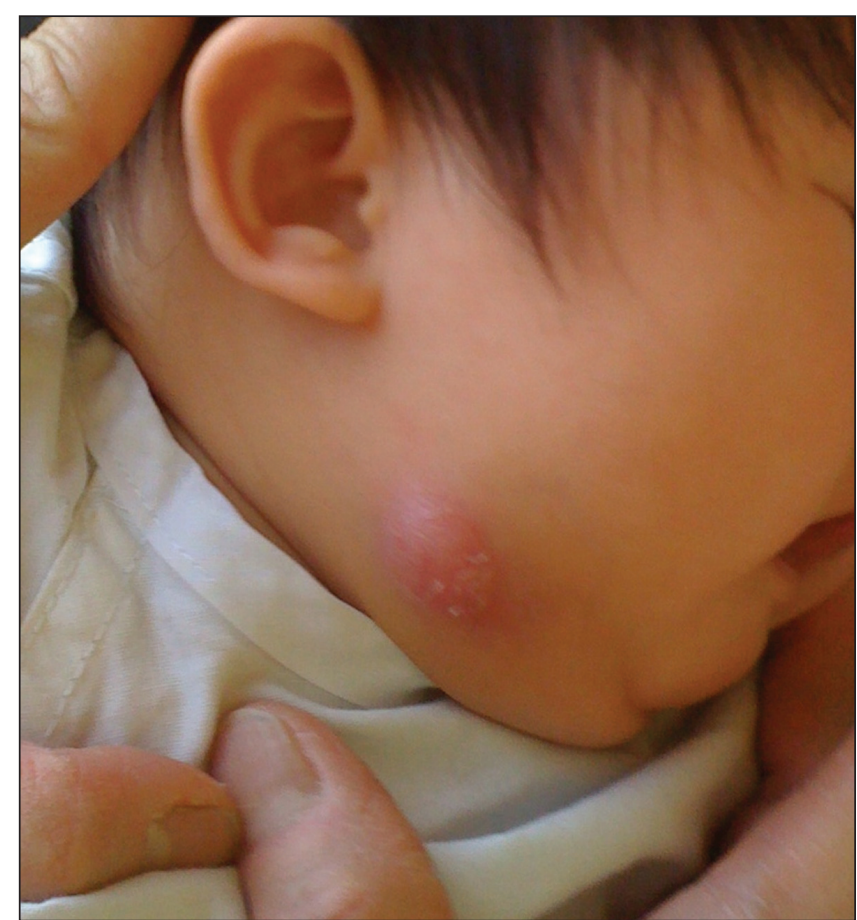

Figure 1) Photo of the patient before incision and drainage of the lesion

incubation, cultures became positive in the liquid Mycobacteria Growth Indicator Tube (BD, USA) mycobacterial detection system. Molecular testing confirmed the presence of $\mathrm{M}$ haemophilum.

Testing for immunological evidence of MTB infection, using the QuantiFERON-TB Gold in Tube (QFT-GIT; Cellestis, Ltd, Australia) interferon-gamma release assay (IGRA) was negative $(0 \mathrm{IU} / \mathrm{mL})$.

\section{DISCUSSION}

Nontuberculous mycobacterial (NTM) adenitis in children has historically been diagnosed using a combination of clinical suspicion, TST and mycobacterial culture (which generally takes weeks to provide results) (2). Treatment is therefore empirical and based on TST and clinical presentation, in the context of possible exposure to MTB. Treatment of NTM adenitis usually involves surgical excision of lesions, unless risk of injury to adjacent structures is high, in which case antibiotic treatment with possible later surgical intervention is the norm (3). Treatment of MTB adenitis involves antimycobacterials.

MAC currently accounts for up to $80 \%$ of NTM adenitis in children (3). Studies involving MAC as the predominant isolate have suggested that a TST induration of $5 \mathrm{~mm}$ to $9 \mathrm{~mm}$ is consistent with NTM adenitis, while indurations $>15 \mathrm{~mm}$ suggest MTB infection (4). Recently, M haemophilum, which is prevalent in the environment and linked with water exposure (5), has been described in Israel and Europe as being responsible for a significant proportion of NTM adenitis in children $(5,6)$. This may be related to recent improvements in culturing techniques allowing detection of this organism (7).

${ }^{1}$ Department of Internal Medicine, Division of Infectious Disease; ${ }^{2}$ Department of Family Medicine; ${ }^{3}$ Department of Pathology and Laboratory Medicine, University of British Columbia; ${ }^{4}$ British Columbia Centre for Disease Control; ${ }^{5}$ Department of Pediatrics, Division of Infectious 8 Immunological Diseases, University of British Columbia, Vancouver, British Columbia

Correspondence: Dr Melanie CM Murray, B432 - 4500 Oak Street, Vancouver, British Columbia V6H 3N1. Telephone 604-875-2212,

fax 604-875-3063, e-mail mel@teetaert.com 
Unlike MAC, M haemophilum infection is associated with greater induration on TST (6), making it difficult to differentiate NTM and MTB infection. QFT-GIT and other region of difference-1 based IGRA tests are more specific for MTB versus TST (8). IGRAs do not cross-react with most NTM, including MAC and M haemophilum and, thus, make useful adjuncts in the evaluation of mycobacterial adenitis (9). However, IGRA tests may have reduced sensitivity in very young children and may not be sufficient on their own to exclude MTB infection (10).

The differential diagnosis of cervical adenitis should always include NTM and MTB as possible causative agents. Sampling of affected lymph nodes for culture and molecular diagnostic testing is crucial. Where suspicion of MTB exists, molecular testing may yield a diagnosis weeks before traditional culture methods, potentially saving children toxicity from antimycobacterial drugs. Culturing of samples is likewise essential for obtaining drug sensitivities, particularly in MTB infection. Unfortunately, the only laboratories routinely using molecular typing of NTM adenitis in Canada are the British Columbia Centre for Disease Control in Vancouver, British Columbia, and the National Microbiology Labs in Winnipeg, Manitoba.

The present patient was at high risk for tuberculosis adenitis (based on history, clinical picture, surgical sample positivity for acid-fast bacilli and TST results), and was initiated on quadruple tuberculosis therapy (ie, isoniazid, rifampin, ethambutol and pyrazinamide).

\section{REFERENCES}

1. McNabb A, Eisler D, Amos M, et al. Assessment of partial sequencing of the 65-kilodalton heat shock protein gene (hsp65) for routine identification of Mycobacterium species isolated from clinical sources. J Clin Microbiol 2004:42:3000-11.

2. Amir J. Non-tuberculous mycobacterial lymphadenitis in children: Diagnosis and management. Isr Med Assoc J 2010;12:49-52.

3. Griffith DE, Aksamit T, Brown-Elliot BA, et al. American Thoracic Society; Infectious Disease Society of America. An official ATS/ IDSA statement: Diagnosis, treatment, and prevention of nontuberculous mycobacterial diseases. Am J Respir Crit Care Med 2007; 175:367-416.

4. Starke JR, Correa AG. Management of mycobacterial infection and disease in children. Pediatr Infect Dis J 1995;14:455-69.

5. Lindeboom JA, Prins JM, Bruijnesteijn van Coppenraet ES, Lindeboom R, Kuijper EJ. Cervicofacial lymphadenitis in children caused by Mycobacterium haemophilum. Clin Infect Dis 2005:41:1569-75. Epub 2005 Oct 28.

6. Cohen YH, Amir J, Ashkenazi S, et al. Mycobacterium haemophilum and lymphadenitis in immunocompetent children, Israel. Emerg Infect Dis 2008:1437-9.
Polymerase chain reaction and sequencing results became available one week later, identifying the acid-fast bacilli as M haemophilum. Medications were changed to ciprofloxacin and rifampin 30 days before mycobacterial cultures identified the organism. This combination provided coverage of $\mathrm{M}$ haemophilum based on the literature (11), and tuberculosis prophylaxis in case of exposure. Due to the location, size and complexity of the masses, antibiotic treatment was chosen to reduce mass size before complete surgical excision, to be performed three months later. The temple mass resolved with antibiotic treatment alone. The patient is currently doing well.

FINANCIAL SUPPORT AND DISCLOSURES: No financial support was obtained for this article. All authors declare no conflicts of interest. TK is supported in part by a Career Award in the Biomedical Sciences from the Burroughs Wellcome Fund and by a CIHR Training Grant from the Canadian Child Health Clinician Scientist Program, in partnership with Sick Kids Foundation, Child \& Family Research Institute (British Columbia), Women \& Children's Health Research Institute (Alberta) and the Manitoba Institute of Child Health.

AUTHOR CONTRIBUTIONS: MCMM drafted the manuscript. JC, PT, JH and TK critically revised the manuscript for content. All authors saw and approved the final manuscript.

7. Ryan CG, Dwyer BW. New characteristics of Mycobacterium haemophilum. J Clin Microbiol 1983;18:976-7.

8. Pai M, Zwerling A, Menzies D. Systematic review: T-cell based assays for the diagnosis of latent tuberculosis infection: An update. Ann Intern Med 2008;149:177-84.

9. Detjen AK, Keil T, Roll S, et al. Interferon-gamma release assays improve the diagnosis of tuberculosis and nontuberculous mycobacterial disease in children in a country with a low incidence of tuberculosis. Clin Infect Dis 2007;45(3):322-8.

10. Mazure M, Jereb J, Vernon A, LoBue P, Goldberg S, Castro K: IGRA Expert Committee; Centers for Disease Control and Prevention (CDC). Updated guidelines for using interferon gamma release assays to detect Mycobacterium tuberculosis infection United States, 2010. MMWR Recomm Rep 2010;59:1-25.

11. Watt B. In-vitro sensitivities and treatment of less common mycobacteria. J Antimicrob Chemother 1997;39:567-74. 


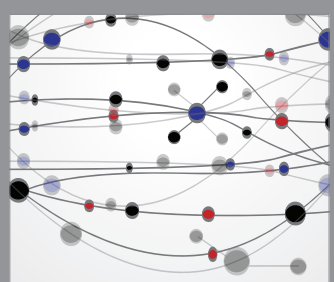

The Scientific World Journal
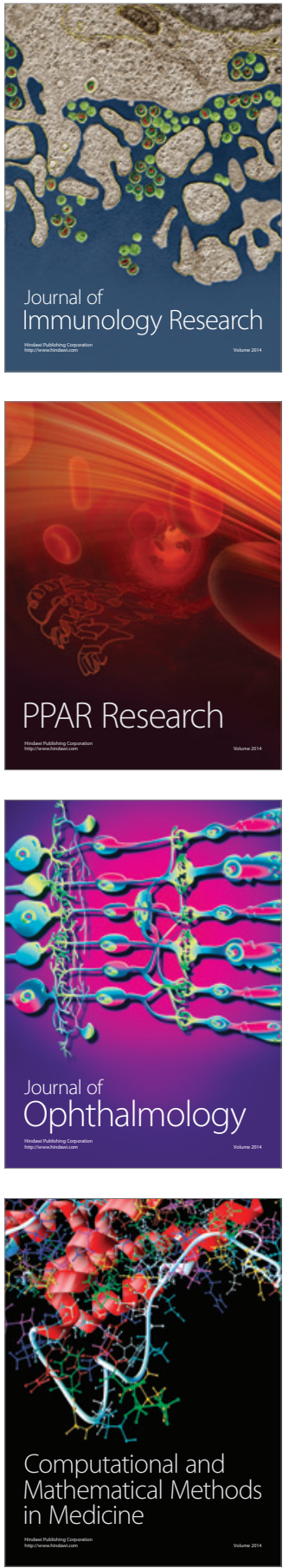

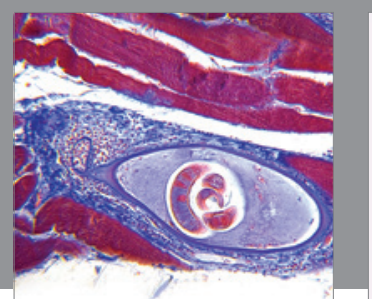

Gastroenterology Research and Practice

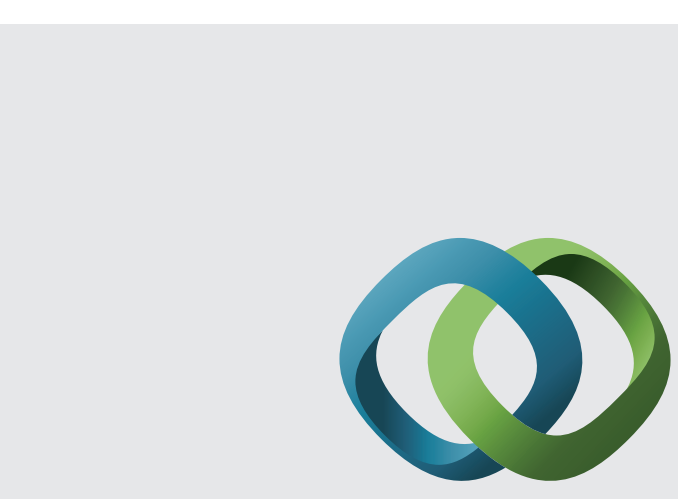

\section{Hindawi}

Submit your manuscripts at

http://www.hindawi.com
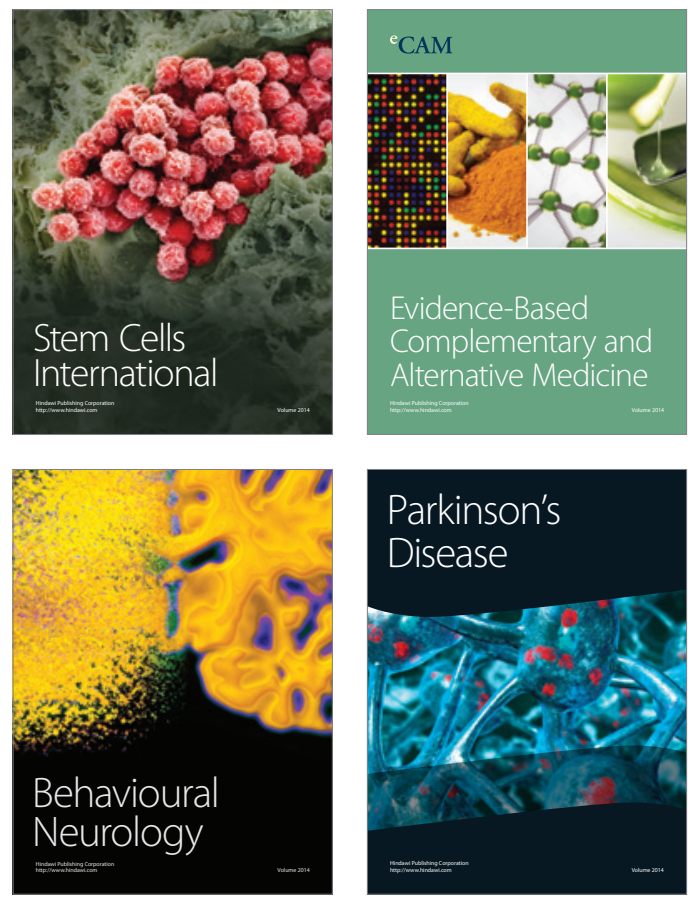
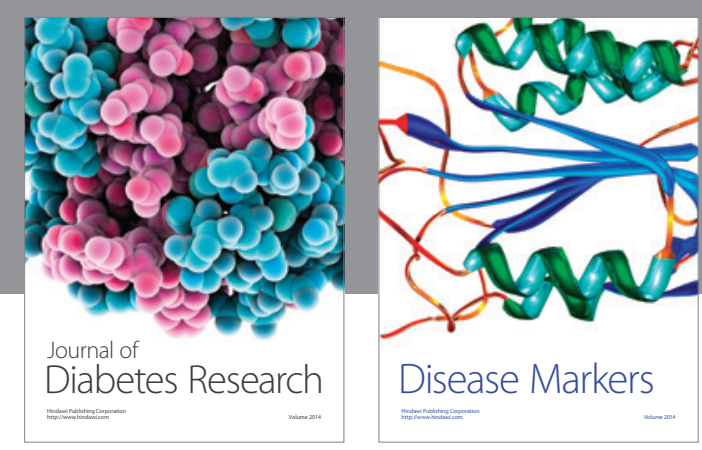

Disease Markers
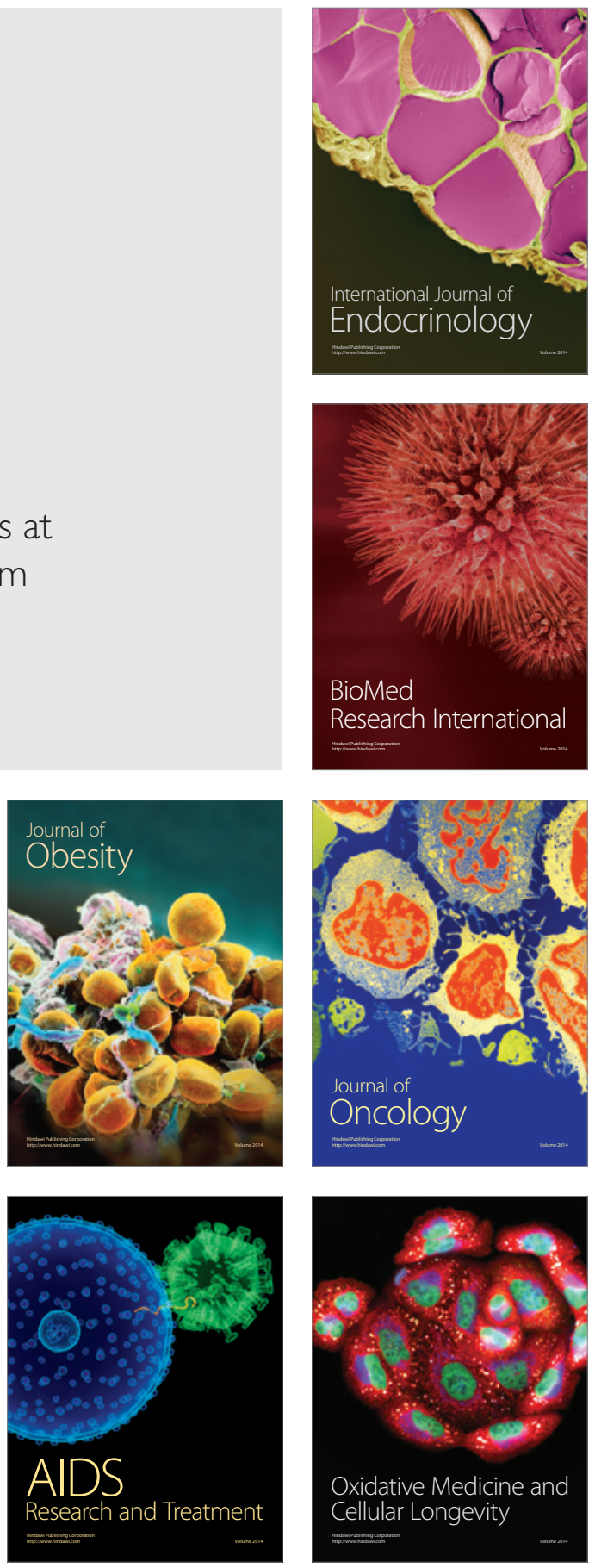